\title{
Noncommutative Topological Entropy of Endomorphisms of Cuntz Algebras II
}

\author{
by
}

\author{
Adam SKALSKI
}

\begin{abstract}
A study of noncommutative topological entropy of gauge invariant endomorphisms of Cuntz algebras begun in our earlier work with J. Zacharias is continued and extended to endomorphisms which are not necessarily of permutation type. In particular it is shown that if $\mathrm{H}$ is an $N$-dimensional Hilbert space, $V$ is an irreducible multiplicative unitary on $\mathrm{H} \otimes \mathrm{H}$ and $F: \mathrm{H} \otimes \mathrm{H} \rightarrow \mathrm{H} \otimes \mathrm{H}$ is the tensor flip, then the Voiculescu entropy of Longo's canonical endomorphism $\rho_{V F} \in \operatorname{End}\left(\mathcal{O}_{N}\right)$ is equal to $\log N$.
\end{abstract}

2010 Mathematics Subject Classification: Primary 46L55, Secondary 37B40.

Keywords: noncommutative topological entropy, Cuntz algebra, polynomial endomorphisms.

\section{Introduction}

Noncommutative topological entropy for transformations of $C^{*}$-algebras introduced by D. Voiculescu in [Voi] is an interesting invariant generalising the classical topological entropy of continuous transformations of compact spaces $([\mathrm{NeS}])$; its value for an endomorphism $\rho$ will be denoted by ht $(\rho)$. The class of transformations most studied from the point of view of the Voiculescu entropy is the family of various noncommutative generalisations of classical shifts, in particular the canonical shift on the Cuntz algebra $\mathcal{O}_{N}$. In [SkZ] together with J.Zacharias we computed the Voiculescu entropy of certain permutation endomorphisms of $\mathcal{O}_{N}$ generalising the canonical shift. Permutation endomorphisms are endomorphisms of $\mathcal{O}_{N}$ associated to so-called permutation unitaries; their properties imply that they can be studied via combinatorial methods.

Communicated by H. Okamoto. Received February 15, 2010. Revised June 10, 2010.

A. Skalski: Department of Mathematics and Statistics,

Lancaster University, Lancaster, LA1 4YF, UK;

current address: Institute of Mathematics, Polish Academy of Sciences, Śniadeckich 8, 00-956 Warszawa, Poland;

e-mail: a.skalski@impan.pl 
The basic method of obtaining lower estimates for the entropy $h t(\rho)$ of a given endomorphism $\rho$ of a $C^{*}$-algebra $\mathrm{A}$ is based on finding a commutative $C^{*}$-subalgebra left invariant by $\rho$ on which $\rho$ is induced by a classical transformation whose entropy can be computed by an application of standard dynamical systems' techniques. The examples of bitstream shifts studied in [NeS] show that it can happen that

$$
\begin{aligned}
\operatorname{ht}(\rho) & >\operatorname{ht}_{c}(\rho) \\
& =\sup \left\{\operatorname{ht}\left(\left.\rho\right|_{\mathrm{C}}\right): \mathrm{C} \text { is a commutative } \rho \text {-invariant } C^{*} \text {-subalgebra of } \mathrm{A}\right\}
\end{aligned}
$$

(see [Ska]). Each permutation endomorphism of $\mathcal{O}_{N}$ leaves invariant the so-called canonical masa (maximal abelian subalgebra) $\mathcal{C}_{N} \subset \mathcal{O}_{N}$ (which can be thought of as a natural 'diagonal' algebra inside $\left.\mathcal{O}_{N}\right)$. Although the analysis in [SkZ] showed that there exist examples when $\operatorname{ht}(\rho)>\operatorname{ht}\left(\left.\rho\right|_{\mathcal{C}_{N}}\right)$, in the cases studied in that paper we still had $h t(\rho)=\operatorname{ht}_{c}(\rho)$ and the actual value of entropy was achieved already on some $\rho$-invariant standard masa (i.e. a masa arising from $\mathcal{C}_{N}$ by a change of coordinates). Moreover the only values of entropy obtained explicitly for endomorphisms of $\mathcal{O}_{N}$ were equal to $\log k$, where $k \in \mathbb{N}$.

In view of the above discussion it is interesting to investigate on one hand the existence of invariant standard masas for endomorphisms of a Cuntz algebra and on the other to seek new ways of establishing lower bounds for Voiculescu entropy for such endomorphisms. The first problem was studied by J. H. Hong, W. Szymański and the author in the recent paper [HSS]. The current work is devoted to making progress in the second. The way forward was suggested by connections between the values of the index of permutation endomorphisms and their entropy discussed in $\left[\mathrm{CS}_{2}\right]$ and resembling the connections between the index of a subfactor of a finite factor and the Connes-Størmer entropy of a canonical shift of Ocneanu (see for example [Hia]).

We begin by introducing the notation and recalling basic statements needed in what follows. In Section 2 we analyse a class of examples of endomorphisms of $\mathcal{O}_{N}$ introduced by M. Izumi in [Izu]. Each endomorphism $\rho$ in this class, although not a permutation endomorphism itself, is at the same time a square root of a permutation endomorphism and the composition of a permutation endomorphism with a Bogolyubov automorphism $\beta$. Moreover $\operatorname{ht}(\rho)=\frac{1}{2} \log N$ and $\operatorname{ht}(\rho \circ \beta)=\log N$ (note that in [HSS] it was shown that when $N=2$ the corresponding endomorphism leaves no standard masa invariant). In Section 3 we prove that the computation of the entropy of Izumi's examples can be interpreted as a special instance of a general fact: each irreducible multiplicative unitary $V$ on a finite-dimensional Hilbert space $\mathrm{H}([\mathrm{BaS}])$ leads to an endomorphism of a corresponding Cuntz algebra whose Voiculescu entropy is equal to the logarithm of the dimension of $\mathrm{H}$. 


\section{$\S 1$. Notation and preliminaries}

Let $N \in \mathbb{N}$ and let $\mathcal{O}_{N}$ denote the Cuntz algebra, i.e. the $C^{*}$-algebra generated by $N$ isometries with orthogonal ranges summing to $1\left(\left[\mathrm{Cu}_{1}\right]\right)$. The isometries generating $\mathcal{O}_{N}$ will be usually denoted by $S_{1}, \ldots, S_{N}$; composition of several generating isometries will be expressed via a multi-index type notation (see for example [SkZ]). For each $k \in \mathbb{N}$ we denote the set of multi-indices of length $k$ by $\mathcal{J}_{k}:=\left\{\left(j_{1}, \ldots, j_{k}\right): j_{1}, \ldots, j_{k} \in\{1, \ldots, N\}\right\}$ and put $\mathcal{J}=\bigcup_{k \in \mathbb{N}} \mathcal{J}_{k}$. There is a well-known 1-1 correspondence between the unital endomorphisms of $\mathcal{O}_{N}$ (denoted further by $\left.\operatorname{End}\left(\mathcal{O}_{N}\right)\right)$ and unitaries in $\mathcal{O}_{N}$, first observed in $\left[\mathrm{Cu}_{2}\right]$ : given $U \in \mathcal{U}\left(\mathcal{O}_{N}\right)$ the associated endomorphism is determined by

$$
\rho_{U}\left(S_{i}\right)=U S_{i}, \quad i=1, \ldots, N
$$

and conversely given $\rho \in \operatorname{End}\left(\mathcal{O}_{N}\right)$ its associated unitary is given by

$$
U_{\rho}=\sum_{i=1}^{n} \rho\left(S_{i}\right) S_{i}^{*} .
$$

If a unitary $U \in \mathcal{O}_{N}$ is a linear combination of elements of the form $S_{i} S_{j}^{*}(i, j=$ $1, \ldots, N)$, the associated transformation $\rho_{U}$ is an automorphism, called a $B o-$ golyubov automorphism (its action corresponds to the change of coordinates in the Hilbert space $\mathbb{C}^{N}$ underlying the Cuntz algebra in the approach due to R. Longo and J. Roberts). For Bogolyubov automorphisms we will usually write $\alpha_{U}$ instead of $\rho_{U}$. In particular the family of automorphisms $\left\{\alpha_{t}: t \in \mathbb{T}\right\}$ provides an action of $\mathbb{T}$ on $\mathcal{O}_{N}$, called the gauge action. The fixed point space of this action is denoted by $\mathcal{F}_{N}$ and is generated as a normed space by the union $\bigcup_{k \in \mathbb{N}} \mathcal{F}_{N}^{k}$, where $\mathcal{F}_{N}^{k}=\operatorname{Lin}\left\{S_{J} S_{K}^{*}: J, K \in \mathcal{J}_{k}\right\}$. It is easy to check that $\mathcal{F}_{N}^{k}$ is isomorphic to $M_{N^{k}} \approx M_{N}^{\otimes k}$ and the corresponding embeddings $\mathcal{F}_{N}^{k} \subset \mathcal{F}_{N}^{k+1}$ are compatible with the usual unital embeddings on the matrix level, so that $\mathcal{F}_{N}$ is a UHF algebra of type $N^{\infty}$. The canonical masa (maximal abelian subalgebra) in $\mathcal{O}_{N}$ is the algebra $\mathcal{C}_{N}$ generated by $\left\{S_{I} S_{I}^{*}: I \in \mathcal{J}\right\}$; a standard masa is a $C^{*}$-subalgebra of $\mathcal{O}_{N}$ equal to $\alpha\left(\mathcal{C}_{N}\right)$ for some Bogolyubov automorphism $\alpha$. Every standard masa is isomorphic to the algebra of continuous functions on $\mathfrak{C}$, the full shift on $N$ letters ([Wal]).

An interesting class of permutation endomorphisms of $\mathcal{O}_{N}$ having a relatively simple combinatorial description was introduced in [Kaw] and was subsequently studied for example in $[\mathrm{Szy}]$ and $\left[\mathrm{CS}_{1}\right]$. Let $\mathcal{P}_{k}$ denote the set of all permutations of the set $\mathcal{J}_{k}$. The permutation endomorphism given by $\sigma \in \mathcal{P}_{k}$ is the endomorphism associated to a unitary $U_{\sigma}=\sum_{J \in \mathcal{J}_{k}} S_{\sigma(J)} S_{J}^{*}$. The canonical shift on $\mathcal{O}_{N}$ is the 
permutation endomorphism associated with the flip unitary $F=\sum_{i, j=1}^{N} S_{i} S_{j} S_{i}^{*} S_{j}^{*}$ and denoted further by $\Phi$.

The standard topological entropy of a continuous transformation $T$ of a compact space ([Wal]) will be denoted by $h_{\mathrm{top}}(T)$. For the definition and basic properties of Voiculescu's noncommutative topological entropy of an endomorphism (or a completely positive map) of a (nuclear) $C^{*}$-algebra we refer to the original paper [Voi] or to the monograph [NeS]. All the information needed to read this note can also be found in [SkZ].

\section{§2. Entropy of an endomorphism coming from a real sector- the square root of a canonical endomorphism}

In [Izu] M. Izumi studied certain explicit examples of endomorphisms of Cuntz algebras motivated by the subfactor theory. One class of them (Example 3.7 of [Izu]) was constructed in the following way: Let $G$ be a finite abelian group of cardinality $N \geq 2$ with the (symmetric) duality bracket $\langle\cdot, \cdot\rangle: G \times G \rightarrow \mathbb{T}$ satisfying the usual conditions $\left(g, g^{\prime}, h \in G\right)$

$$
\begin{gathered}
\overline{\langle g, h\rangle}=\langle-g, h\rangle, \quad \sum_{h \in G}\langle h, g\rangle= \begin{cases}0 & \text { if } g \neq e, \\
N & \text { if } g=e,\end{cases} \\
\langle g, h\rangle\left\langle g^{\prime}, h\right\rangle=\left\langle g+g^{\prime}, h\right\rangle, \quad\langle g, h\rangle=\langle h, g\rangle
\end{gathered}
$$

(the group operation in $G$ will be written additively). If $G=\mathbb{Z} / N \mathbb{Z}$ one can put $\langle k, l\rangle:=\exp (2 \pi i(k l) / N)$. We will use elements of $G$ as indices of generating isometries in $\mathcal{O}_{N}$. Define unitaries $U(g) \in \mathcal{F}_{N} \subset \mathcal{O}_{N}(g \in G)$ by

$$
U(g)=\sum_{h \in G}\langle g, h\rangle S_{h} S_{h}^{*},
$$

and the endomorphism $\rho \in \operatorname{End}\left(\mathcal{O}_{N}\right)$ by

$$
\rho\left(S_{g}\right)=\frac{1}{\sqrt{N}} \sum_{h \in G}\langle g, h\rangle S_{h} U(g)^{*} .
$$

The endomorphism $\rho$ was studied in detail in [HSS]. It does not leave $\mathcal{C}_{N}$ invariant. The unitary associated with $\rho$ is equal to

$$
V=\frac{1}{\sqrt{N}} \sum_{g, h, l \in G}\langle g, h-l\rangle S_{h} S_{l} S_{l}^{*} S_{g}^{*}
$$

Define, for each $h \in G$,

$$
\widetilde{S}_{h}=\frac{1}{\sqrt{N}} \sum_{a \in G}\langle h, a\rangle S_{a}
$$


and let $\beta \in \operatorname{Aut}\left(\mathcal{O}_{N}\right)$ be given by

$$
\beta\left(S_{h}\right)=\widetilde{S}_{h}, \quad h \in G .
$$

It is easy to see that $\beta$ is a Bogolyubov automorphism.

Theorem 2.1. The endomorphism $\rho^{\prime}:=\rho \circ \beta$ is a permutation endomorphism of $\mathcal{O}_{N}$ given by the formula (notation as above)

$$
\rho^{\prime}\left(S_{h}\right)=\sum_{g \in G} S_{g} S_{h+g} S_{h+g}^{*}, \quad h \in G .
$$

Moreover $\operatorname{ht}\left(\rho^{\prime}\right)=\log N$.

Proof. The first statement is shown in [HSS]. As the endomorphism $\rho^{\prime}$ is induced by a unitary in $\mathcal{F}_{N}^{2}$, Theorem 2.2 of [SkZ] implies that $\operatorname{ht}\left(\rho^{\prime}\right) \leq \log N$. As $\rho^{\prime}$ is a permutation endomorphism, it leaves $\mathcal{C}_{N}$ invariant and $\left.\rho^{\prime}\right|_{\mathcal{C}_{N}}$ is induced by a continuous transformation $T_{\rho^{\prime}}$ of $\mathfrak{C}$. Note that we have (for $h, k \in G$ )

$$
\begin{gathered}
\rho^{\prime}\left(S_{h} S_{h}^{*}\right)=\sum_{g \in G} S_{g} S_{h+g} S_{h+g}^{*} S_{g}^{*}, \\
\rho^{\prime}\left(S_{h} S_{k} S_{k}^{*} S_{h}^{*}\right)=\sum_{g \in G} S_{g} S_{h+g} S_{k+h+g} S_{k+h+g}^{*} S_{h+g}^{*} S_{g}^{*},
\end{gathered}
$$

and so on. The analysis of the transformations on cylinder sets (see [SkZ] or [Szy]) implies that $T_{\rho^{\prime}}$ is given by the formula

$$
\left(T_{\rho^{\prime}}(w)\right)_{k}=w_{k+1}-w_{k}, \quad w:=\left(w_{n}\right)_{n=1}^{\infty} \in \mathfrak{C}, k \in \mathbb{N} .
$$

Hence an application of an extension of Lemma 3.2 of [SkZ] (although this lemma was stated in [SkZ] only for $N=2$, it is easy to see that its formulation and proof can be easily adapted to arbitrary $N)$ shows that $h_{\text {top }}\left(T_{\rho^{\prime}}\right)=\log N$ so that $\operatorname{ht}\left(\rho^{\prime}\right) \geq \operatorname{ht}\left(\left.\rho^{\prime}\right|_{\mathcal{C}_{N}}\right)=h_{\text {top }}\left(T_{\rho^{\prime}}\right)=\log N$.

Theorem 2.2. The Voiculescu entropy of $\rho$ is equal to $\frac{1}{2} \log N$.

Proof. It is easily checked that $\operatorname{ht}(\rho)=\frac{1}{2} \mathrm{ht}\left(\rho^{2}\right)$. Write $\gamma:=\rho^{2}$. It suffices to show that $\operatorname{ht}(\gamma)=\log N$. This will follow from the general result in Theorem 3.2, but here we can provide a direct proof, as $\gamma$ is a permutation endomorphism given by the formula $(g \in G)$

$$
\gamma\left(S_{g}\right)=\sum_{k \in G} S_{k} S_{g+k} S_{k}^{*}
$$

(see [HSS]). As the associated unitary $V_{\gamma}:=\sum_{g, h \in G} S_{g} S_{h+g} S_{g}^{*} S_{h}^{*}$ belongs to $\mathcal{F}_{N}^{2}$, Theorem 2.2 of [SkZ] implies that $\operatorname{ht}(\gamma) \leq \log N$. 
Examine the action of $\gamma$ on $\mathcal{C}_{N}$. For each $n \in \mathbb{N}$ and $g_{1}, \ldots, g_{n} \in G$,

$$
\gamma\left(S_{g_{1}} \cdots S_{g_{n}} S_{g_{n}}^{*} \cdots S_{g_{1}}^{*}\right)=\sum_{h \in G} S_{h} S_{g_{1}+h} \cdots S_{g_{n}+h} S_{g_{n}+h}^{*} \cdots S_{g_{1}+h}^{*} S_{h}^{*}
$$

so that once more analysing the cylinder sets we see that $\left.\gamma\right|_{\mathcal{C}_{N}}$ is induced by the continuous transformation defined by

$$
\left(T_{\gamma}(w)\right)_{k}=w_{1}+w_{k+1}, \quad w=\left(w_{n}\right)_{n=1}^{\infty} \in \mathfrak{C}, k \in \mathbb{N} .
$$

Thus appealing again to a suitable generalisation of Lemma 3.2 in [SkZ] yields $\operatorname{ht}\left(\gamma \mid \mathcal{C}_{N}\right)=h_{\text {top }}\left(T_{\gamma}\right)=\log N$. This ends the proof.

Note that Theorems 2.1 and 2.2 yield an example of an endomorphism $\rho$ of $\mathcal{O}_{N}$ and a Bogolyubov automorphism $\beta$ such that ht $(\rho \circ \beta) \neq \operatorname{ht}(\rho)$ (although, as follows from [DyS], the Voiculescu entropy of each Bogolyubov automorphism of $\mathcal{O}_{N}$ is 0$)$.

Let $G=\mathbb{Z} / 2 \mathbb{Z}=\{0,1\}$ with the natural duality bracket $(\langle 1,1\rangle=-1$, all other brackets take value 1$)$. The corresponding endomorphism in the class discussed above is then given by

$$
\rho\left(S_{0}\right)=\frac{1}{\sqrt{2}}\left(S_{0}+S_{1}\right), \quad \rho\left(S_{0}\right)=\frac{1}{\sqrt{2}}\left(S_{0} S_{0} S_{0}^{*}+S_{1} S_{1} S_{1}^{*}-S_{1} S_{0} S_{0}^{*}-S_{0} S_{1} S_{1}^{*}\right)
$$

In Section 6 of [HSS] we showed that $\rho$ defined via the formulas in (2.2) does not leave any standard masa invariant. This naturally leads to the following closely connected questions: can one characterise those masas in $\mathcal{O}_{N}$ left invariant by $\rho$ ? Do we have ht $(\rho)=\operatorname{ht}_{c}(\rho)$ ?

It would be very interesting to investigate the entropy of other examples of real sectors given in [Izu]. This would require completely new methods even to obtain upper estimates, as Theorem 2.2 of [SkZ] applies only to the endomorphisms associated to unitaries in $\mathcal{F}_{N}$, and other examples of Section 3 of [Izu] are not of this type.

\section{§3. Entropy of canonical endomorphisms associated to multiplicative unitaries}

Consider again the endomorphism $\rho$ associated to the unitary defined in (2.1) via a symmetric duality bracket on a finite abelian group $G$ discussed in the last section. Let $\tau$ be the faithful trace on $\mathcal{F}_{N}$ and let $\phi=\tau \circ E$, where $E: \mathcal{O}_{N} \rightarrow \mathcal{F}_{N}$ is the canonical conditional expectation (given by integrating the gauge action). It follows from Lemma 2.1 of $\left[\mathrm{Lo}_{2}\right]$ that the endomorphism $\rho$ preserves $\phi$, so it also extends to an endomorphism of $\mathrm{M}:=\pi_{\phi}\left(\mathcal{O}_{N}\right)^{\prime \prime}$, where $\pi_{\phi}$ denotes the GNS 
representation with respect to $\phi$ (we will denote the extension by $\widetilde{\rho}$ ). It follows from the easily checked condition in Corollary 4.3 of $[\mathrm{CoP}]$ that $\tilde{\rho}$ is irreducible, i.e. $\widetilde{\rho}(\mathrm{M})^{\prime} \cap \mathrm{M}=\mathbb{C} I_{\mathrm{M}}$.

As discussed in Section 2, in [Izu] it is observed (as a consequence of results in $\left[\mathrm{Lo}_{1}\right]$ and [Wat]) that $\rho$ is a restriction of a 'square root' of a canonical endomorphism of the $I I I_{1 / N}$-factor generated by $\mathcal{O}_{N}$ in the GNS representation with respect to the state $\phi$. It follows from Proposition 2.5 in [Izu] that if we consider the conditional expectation $E_{\rho}: \mathcal{O}_{N} \rightarrow \rho\left(\mathcal{O}_{N}\right)$ defined by

$$
E_{\rho}(x)=\rho\left(S_{e}^{*} \rho(x) S_{e}\right),
$$

where $e \in G$ is the neutral element, then Ind $E_{\rho}=N$ and it is a minimal index in the sense of Hiai (see [Kos] and references therein). Moreover the endomorphism $\gamma=\rho^{2}$ is related to the left regular representation of the group $G$. Indeed, it is easy to check that

$$
\gamma^{2}=\Phi \circ \gamma
$$

which due to Proposition 2.1 of $\left[\mathrm{Cu}_{3}\right]$ is equivalent to the fact that the unitary associated with $\gamma$ is a product of a multiplicative unitary on $\mathbb{C}^{n} \otimes \mathbb{C}^{n}([\mathrm{BaS}])$ and the flip unitary $F=\sum_{g, h \in G} S_{h} S_{g} S_{h}^{*} S_{g}^{*}$.

As suggested by the above discussion, there is a connection between the entropy computation in Section 2, index values of the inclusion $\widetilde{\rho}(\mathrm{M}) \subset \mathrm{M}$ and the fact that $\rho^{2}$ is related to the left regular representation of a finite group. Indeed, Theorem 2.2 may be viewed as a special instance of a general entropy result related to interaction between finite-dimensional Kac algebras and index for subfactors stated in Theorem 3.2 below. Recall two basic definitions from [BaS]:

Definition 3.1. Let $\mathrm{H}$ be a Hilbert space and let $\Sigma$ denote the unitary tensor flip in $B(\mathrm{H} \otimes \mathrm{H})$. A unitary $V \in B(\mathrm{H} \otimes \mathbf{H})$ is called a multiplicative unitary if it satisfies the pentagonal equation

$$
V_{12} V_{13} V_{23}=V_{23} V_{12}
$$

(we use the standard leg notation, so that the operators in the equality above act on $\mathrm{H} \otimes \mathrm{H} \otimes \mathrm{H})$. A multiplicative unitary $V \in B(\mathrm{H} \otimes \mathrm{H})$ is called irreducible if there exists a self-adjoint unitary $U \in B(\mathrm{H})$ such that $(\Sigma(1 \otimes U) V)^{3}=1$ and both operators $\hat{V}=\Sigma(U \otimes 1) V(U \otimes 1) \Sigma$ and $\tilde{V}=(U \otimes U) \hat{V}(U \otimes U)$ are multiplicative unitaries.

Baaj and Skandalis showed in Section 4 of $[\mathrm{BaS}]$ that if $\mathrm{H}$ is finite-dimensional then each multiplicative unitary in $B(\mathrm{H} \otimes \mathrm{H})$ is irreducible up to multiplicity (i.e. 
each multiplicative unitary on $\mathrm{H}$ is unitarily equivalent to a tensor product of an irreducible one and $1_{K} \otimes 1_{K}$ for some Hilbert space $K$ ). In particular multiplicative unitaries associated in a canonical way with finite-dimensional Kac algebras are irreducible.

Theorem 3.2. Let $V$ be an irreducible multiplicative unitary on $\mathrm{H} \otimes \mathrm{H}$, where $\mathrm{H}$ is an $N$-dimensional Hilbert space; view $V$ as a matrix in $M_{N} \otimes M_{N}$ and further via the usual isomorphism $M_{N} \otimes M_{N} \approx \mathcal{F}_{N}^{2} \subset \mathcal{O}_{N}$ as a unitary in $\mathcal{O}_{N}$. Let $F$ be the flip unitary in $\mathcal{F}_{N}^{2}$. The topological entropy of the endomorphism of $\mathcal{O}_{N}$ associated with $V F$ is equal to $\log N$.

Proof. Denote $R=V F, \gamma:=\rho_{R}$. As $R \in \mathcal{F}_{N}^{2}$, Theorem 2.2 of [SkZ] implies that $\operatorname{ht}(\gamma) \leq \log N$

As mentioned above, Lemma 2.1 of $\left[\mathrm{Lo}_{2}\right]$ implies that $\gamma$ preserves $\phi$, so $\gamma$ extends to an endomorphism of $\mathrm{M}:=\pi_{\phi}\left(\mathcal{O}_{N}\right)^{\prime \prime}$, denoted by $\widetilde{\gamma}$. Irreducibility of $V$ implies that $\widetilde{\gamma}$ is a canonical endomorphism for an irreducible inclusion of factors $\mathrm{N} \subset \mathrm{M}$ (Corollary 4.3 of $\left[\mathrm{Lo}_{2}\right]$ ), where $\mathrm{N}=\{x \in \mathrm{M}: \widetilde{\Phi}(x)=\widetilde{\gamma}(x)\}$ is the fixed point algebra for the coaction associated to $V\left(\left[\mathrm{Lo}_{2}\right],\left[\mathrm{Cu}_{3}\right]\right)$. By Proposition 3.1 in $\left[\mathrm{Lo}_{2}\right], \operatorname{Ind}(\widetilde{\gamma})=N^{2}$.

Let $\mathrm{M}^{(0)}:=\pi_{\phi}\left(\mathcal{F}_{N}\right)^{\prime \prime}=\pi_{\phi}\left(\bigcup_{n \in \mathbb{N}} \mathcal{F}_{N}^{n}\right)^{\prime \prime}$. Due to Proposition 4.7 in $\left[\mathrm{Lo}_{2}\right], \mathrm{M}^{(0)}$ is equal to the strong closure of the algebra $\bigcup_{n \in \mathbb{N}} \widetilde{\gamma}^{n}(\mathrm{M})^{\prime} \cap \mathrm{M}$. As $V \in \mathcal{F}_{N}$, the endomorphism $\widetilde{\gamma}$ leaves $\mathrm{M}^{(0)}$ invariant. By Theorem 3.2.2(ii) in [NeS] we have $\mathrm{h}_{\phi}(\widetilde{\gamma})=\mathrm{h}_{\phi}(\gamma)$, where $h_{\omega}(\alpha)$ denotes the CNT entropy of an endomorphism $\alpha$ preserving a state $\omega$ (see $[\mathrm{NeS}]$ for the precise definitions - although Theorem 3.2.2(ii) is stated for automorphisms, its proof is valid also when $\rho$ is just an endomorphism). As $h t(\gamma) \geq \mathrm{h}_{\phi}(\gamma)$ by Theorem $6.2 .2($ ii) in $[\mathrm{NeS}]$, to finish the proof it suffices to show that $\mathrm{h}_{\phi}(\widetilde{\gamma}) \geq \log N$. Note that as the canonical conditional expectation $E$ extends to a $\phi$-preserving normal conditional expectation $\widetilde{E}: \mathrm{M} \rightarrow \mathrm{M}^{(0)}$, by Theorem 3.2.2(v) in $[\mathrm{NeS}]$ we have $\mathrm{h}_{\phi}(\widetilde{\gamma}) \geq \mathrm{h}_{\phi}\left(\left.\widetilde{\gamma}\right|_{\mathrm{M}^{(0)}}\right)$.

Let $\mathrm{N}^{(0)}:=\mathrm{N} \cap \mathrm{M}^{(0)}$. Note that because both $\gamma$ and the canonical shift $\Phi$ commute with the gauge action, we have $\mathrm{N}^{(0)}=\widetilde{E}(\mathrm{~N})$. As the canonical expectation $E_{\gamma}: \mathrm{M} \rightarrow \widetilde{\gamma}(\mathrm{M})$ discussed in Lemma 4.6 of $\left[\mathrm{Lo}_{2}\right]$ preserves the trace on $\mathrm{M}^{(0)}$, due to Lemma 7.3.5 in [Cho] we see that $\mathrm{N}^{(0)}$ is a subfactor of $\mathrm{M}^{(0)}$; Proposition 7.3.6 of the same paper ${ }^{1}$ implies $\left[\mathrm{M}^{(0)}, \mathrm{N}^{(0)}\right]=(\operatorname{Ind}(\widetilde{\gamma}))^{1 / 2}=N$. Further we can use the observation in Theorem 7.3.7 of [Cho] (see also Corollary 4.3 in [ $\left.\mathrm{Lo}_{2}\right]$ ) that $\left.\widetilde{\gamma}\right|_{M^{(0)}}$ is conjugate to Ocneanu's canonical shift associated with the inclusion $\mathrm{N}^{(0)} \subset \mathrm{M}^{(0)}$; note that now we are in the framework of finite factor inclusions.

\footnotetext{
${ }^{1}$ Note that in Lemma 7.2.1 in [Cho] the operator $V$ should be defined as $V=(1 / \lambda) \gamma(e) f e$ and is only a partial isometry - this does not affect further reasoning and the main results of that paper remain valid.
} 
Corollary 4.6 of [Hia] then gives $\mathrm{h}_{\phi}\left(\left.\widetilde{\gamma}\right|_{\mathrm{M}^{(0)}}\right)=\log \left(\left[\mathrm{M}^{(0)}, \mathrm{N}^{(0)}\right]\right)=\log N$, provided the inclusion $\mathrm{N}^{(0)} \subset \mathrm{M}^{(0)}$ is extremal and strongly amenable. Extremality follows from the equality $\left(\mathrm{N}^{(0)}\right)^{\prime} \cap \mathrm{M}^{(0)}=\mathbb{C} I_{\mathrm{M}}$, which itself is a consequence of Theorem 6.6 in [LoR] and Proposition 3.2 of $\left[\mathrm{Lo}_{2}\right]$. By Theorem 1 in [Pop] strong amenability of the inclusion in question will follow if we can only show that it has finite depth, as $\mathrm{M}^{(0)}$ is a hyperfinite factor. As the Jones tunnel corresponding to the inclusion $\mathrm{N}^{(0)} \subset \mathrm{M}^{(0)}$ is given by

$$
\cdots \subset \widetilde{\gamma}\left(\mathrm{N}^{(0)}\right) \subset \widetilde{\gamma}\left(\mathrm{M}^{(0)}\right) \subset \mathrm{N}^{(0)} \subset \mathrm{M}^{(0)} \subset \cdots
$$

it suffices to show that $\widetilde{\gamma}\left(\mathrm{N}^{(0)}\right)^{\prime} \cap \mathrm{M}^{(0)}$ is a factor. The earlier observation that $\left(\mathrm{N}^{(0)}\right)^{\prime} \cap \mathrm{M}^{(0)}=\mathbb{C} I_{\mathrm{M}}$, the fact that $\widetilde{\gamma}\left(\mathrm{N}^{(0)}\right)^{\prime}=\widetilde{\Phi}\left(\mathrm{N}^{(0)}\right)^{\prime}$ and a suitably adapted argument from Corollary 3.3 of $\left[\mathrm{Lo}_{2}\right]$ furnishes precisely that (one can show that $\left.\widetilde{\gamma}\left(\mathrm{N}^{(0)}\right)^{\prime} \cap \mathrm{M}^{(0)}=\mathcal{F}_{N}^{1}\right)$.

Conceptually the reason for having $\operatorname{ht}\left(\rho_{V F}\right)=\log N$ is that $\rho_{V F}$ is indeed a map closely related to the canonical shift on $\mathcal{O}_{N}$, as is suggested by the formula (3.1); several instances of such analogies can be found in [LoR].

\section{Acknowledgements}

The work on this note was started during a visit of the author to University of Tokyo in October-November 2009 funded by a JSPS Short Term Postdoctoral Fellowship. The author would like to thank the referee for thoughtful comments improving the presentation of the paper.

\section{References}

[BaS] S. Baaj and G. Skandalis, Unitaires multiplicatifs et dualité pour les produits croisés de $C^{*}$-algèbres, Ann. Sci. École Norm. Sup. (4) 26 (1993), 425-488. Zbl 0804.46078 MR 1235438

[Cho] M. Choda, A $C^{*}$-dynamical entropy and applications to canonical endomorphisms, J. Funct. Anal. 173 (2000), 453-480. Zbl 0962.46048 MR 1760622

[CoP] R. Conti and C. Pinzari, Remarks on the index of endomorphisms of Cuntz algebras, J. Funct. Anal. 142 (1996), 369-405. Zbl 0932.46048 MR 1423039

$\left[\mathrm{CS}_{1}\right]$ R. Conti and W. Szymański, Labeled trees and localized automorphisms of the Cuntz algebras, Trans. Amer. Math. Soc. 363 (2011), 5847-5870. MR 2817412

$\left[\mathrm{CS}_{2}\right] \_$, Computing the Jones index of quadratic permutation endomorphisms of $\mathrm{O}_{2}$, J. Math. Phys. 50 (2009), 012705, 5 pp. MR 2492597

$\left[\mathrm{Cu}_{1}\right]$ J. Cuntz, Simple $C^{*}$-algebras generated by isometries, Comm. Math. Phys. 57 (1977), 173-185. Zbl 0399.46045 MR 0467330

$\left[\mathrm{Cu}_{2}\right]$ - Automorphisms of certain simple $C^{*}$-algebras, in Quantum fields-algebras, processes (Bielefeld, 1978), Springer, 1980, 187-196. Zbl 0475.46046 MR 0601811 
$\left[\mathrm{Cu}_{3}\right]$, Regular actions of Hopf algebras on the $C^{*}$-algebra generated by a Hilbert space, in Operator algebras, mathematical physics, and low-dimensional topology (Istanbul, 1991), Res. Notes Math. 5, A K Peters, Wellesley, MA, 1993, 87-100. Zbl 0803.46062 MR 1259060

[DyS] K. Dykema and D. Shlyakhtenko, Exactness of Cuntz-Pimsner $C^{*}$-algebras, Proc. Edinburgh Math. Soc. (2) 44 (2001), 425-444. Zbl 0985.46034 MR 1880402

[Hia] F. Hiai, Entropy for canonical shifts and strong amenability, Int. J. Math. 6 (1995), 381-396. Zbl 0836.46054 MR 1327155

[HSS] J. H. Hong, A. Skalski and W. Szymański, On invariant MASAs for endomorphisms of the Cuntz algebras, Indiana Univ. Math. J., to appear; arXiv:1001.1899.

[Izu] M. Izumi, Subalgebras of infinite $C^{*}$-algebras with finite Watatani indices I. Cuntz algebras, Comm. Math. Phys. 155 (1993), 157-182. Zbl 0803.46066 MR 1228532

[Kaw] K. Kawamura, Polynomial endomorphisms of the Cuntz algebras arising from permutations. I. General theory, Lett. Math. Phys. 71 (2005), 149-158. Zbl 1084.47063 MR 2134694

[Kos] H. Kosaki, Type III factors and index theory, Lecture Notes Ser. 43, Seoul National Univ., Seoul, 1998. Zbl 0915.46053 MR 1662525

$\left[\mathrm{Lo}_{1}\right]$ R. Longo, Index of subfactors and statistics of quantum fields. II. Correspondences, braid group statistics and Jones polynomial, Comm. Math. Phys. 130 (1990), 285-309. Zbl 0705.46038 MR 1059320

$\left[\mathrm{Lo}_{2}\right]$, A duality for Hopf algebras and for subfactors, Comm. Math. Phys. 159 (1994), 133-150. Zbl 0802.46075 MR 1257245

[LoR] R. Longo and J. E. Roberts, A theory of dimension, K-Theory 11 (1997), $103-159$. Zbl 0874.18005 MR 1444286

$[\mathrm{NeS}]$ S. Neshveyev and E. Størmer, Dynamical entropy in operator algebras, Ergeb. Math. Grenzgeb. 50, Springer, Berlin, 2006. Zbl 1109.46002 MR 2251116

[Pop] S. Popa, Classification of amenable subfactors of type II, Acta Math. 172 (1994), 163-255. Zbl 0853.46059 MR 1278111

[Ska] A. Skalski, On automorphisms of $C^{*}$-algebras whose Voiculescu entropy is genuinely noncommutative, Ergodic Theory Dynam. Systems 31 (2011), 951-954. Zbl pre05918102

[SkZ] A. Skalski and J. Zacharias, Noncommutative topological entropy of endomorphisms of Cuntz algebras, Lett. Math. Phys. 86 (2008), 115-134. Zbl 1182.46055 MR 2465749

[Szy] W. Szymański, On localized automorphisms of the Cuntz algebras which preserve the diagonal subalgebra, in New development of operator algebras, RIMS Kôkyûroku 1587 (2008), 109-115.

[Voi] D. Voiculescu, Dynamical approximation entropies and topological entropy in operator algebras, Comm. Math. Phys. 170 (1995), 249-281. Zbl 0824.46079 MR 1334396

[Wal] P. Walters, An introduction to ergodic theory, Grad. Texts in Math. 79, Springer, New York, 1982. Zbl 0475.28009 MR 0648108

[Wat] Y. Watatani, Index for $C^{*}$-subalgebras, Mem. Amer. Math. Soc. 83 (1990), no. 424. Zbl 0697.46024 MR 0996807 